\title{
Two highly reddened young open clusters located beyond the Sagittarius arm $^{\star, \star \star}$
}

\author{
A. E. Piatti ${ }^{1}$ and J. J. Clariáa \\ 1 Instituto de Astronomía y Física del Espacio, CC 67, Suc. 28, 1428, Buenos Aires, Argentina \\ 2 Observatorio Astronómico, Universidad Nacional de Córdoba, Laprida 854, 5000 Córdoba, Argentina \\ Received 10 October 2001 / Accepted 3 April 2002
}

\begin{abstract}
We present the results of CCD BVI Johnson-Cousins photometry down to $V \sim 19$ mag in the regions of the unstudied stellar groups Pismis 23 and $\mathrm{BH} 222$, both projected close to the direction towards the Galactic centre. We measured $V$ magnitude and $B-V$ and $V-I$ colours for a total of 928 stars in fields of about $4^{\prime} \times 4^{\prime}$. Pismis 23 is conclusively a physical system, since a clear main sequence and other meaningful features can be seen in the colour-magnitude diagrams. The reality of this cluster is also supported by star counts carried out within and outside the cluster field. For Pismis 23 we derive colour excesses $E(B-V)=2.0 \pm 0.1$ and $E(V-I)=2.6 \pm 0.1$, a distance from the Sun of $2.6 \pm 0.6 \mathrm{kpc}(Z=-19 \mathrm{pc})$ and an age of $300 \pm 100 \mathrm{Myr}$ (assuming solar metal content). BH 222 appears to be a young open cluster formed by a vertical main sequence and by a conspicuous group of luminous, typically red supergiant stars. We derived for this cluster a colour excess of $E(V-I)=2.4 \pm 0.2$, a distance from the Sun of $6.0 \pm 2.7 \mathrm{kpc}(Z=-46 \mathrm{pc})$ and an age of $60 \pm 30 \mathrm{Myr}$. The resulting reddening and distance estimates place these two young objects among the most reddened and distant open clusters known in the direction towards the Galactic centre. They are located beyond the Sagittarius arm, close to the direction where this arm probably bifurcates into two arms.
\end{abstract}

Key words. open clusters and associations: individual: Pismis 23, BH 222 - open clusters and associations: general - techniques: photometric

\section{Introduction}

Open clusters constitute one of the most important fields of research in observational astronomy. Aside from the intrinsic interest of these objects, the study of the open cluster system lends itself readily to a set of applications in several areas of modern astrophysics, such as the assessment of chemical abundance gradients in the disk (see, e.g., Piatti et al. 1995; Twarog et al. 1997), galactic structure and evolution (e.g., Janes \& Adler 1982; Janes \& Phelps 1994), interactions between thin and thick disks (e.g., Sandage 1988), as well as theories of stellar formation and evolution (e.g., Meynet et al. 1993; Phelps \& Janes 1993).

The open cluster catalogue by Lyngå (1987) includes 1151 entries. However, very little is known for many of them, except their positions and estimates of their angular

Send offprint requests to: A. E. Piatti,

e-mail: andres@iafe.uba.ar

* Based on observations made at the University of Toronto (David Dunlap Observatory) 24-inch telescope, Las Campanas, Chile.

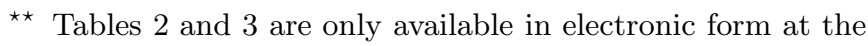
CDS via anonymous ftp to

cdsarc.u-strasbg.fr (130.79.128.5) or via

http://cdsweb.u-strasbg.fr/cgi-bin/qcat?J/A+A/388/179 sizes. The present paper belongs to a series devoted to the study of numerous, mostly unstudied, poorly populated open clusters (or candidates), preferably located towards the central parts of the Galaxy (see, e.g., Piatti \& Clariá 2001a, and references therein). The primary purpose of this systematic survey is to determine whether the observed objects are genuine physical systems or if, on the contrary, the apparent enhancement of stars in a sky region is a consequence of random fluctuations of the stellar density in that zone. Information on the distance, interstellar reddening, age and membership of those objects confirmed as open clusters, is obtained through the analysis of their colour-magnitude diagrams (CMDs). In particular, open clusters located towards the Galactic centre play an important role in that they provide the advantage of tracing the structure and evolution of the inner disk more precisely. Many of these objects, however, have not been studied in detail yet because they are either relatively faint objects or located in obscured and/or crowded fields. This is the case of the southern open cluster candidates Pismis $23\left(\alpha_{2000}=16^{\mathrm{h}} 23^{\mathrm{m}} 58^{\mathrm{s}}\right.$, $\left.\delta_{2000}=-48^{\circ} 53^{\prime} 33^{\prime \prime} ; l=334^{\circ} 66, b=-0^{\circ} 42\right)$ and $\mathrm{BH} 222$ $\left(\alpha_{2000}=17^{\mathrm{h}} 18^{\mathrm{m}} 47^{\mathrm{s}}, \delta_{2000}=-38^{\circ} 17^{\prime} 22^{\prime \prime} ; l=3499^{\circ} 12\right.$, $b=-0.44)$, both projected close to the Galactic centre. Pismis 23, also known as Lyngå 10 (Lyngå 1965), OCL-967 
Table 1. Journal of observations.

\begin{tabular}{lcccc}
\hline \hline Cluster & $\begin{array}{c}\text { Date } \\
\text { (UT) }\end{array}$ & Filter & Exposures & $\begin{array}{c}\text { seeing } \\
\left({ }^{\prime \prime}\right)\end{array}$ \\
\hline Pismis 23 & 1995 June 30 & $B$ & $1 \times 60 \mathrm{~s}, 2 \times 900 \mathrm{~s}$ & 1.5 \\
& & $V$ & $1 \times 60 \mathrm{~s}, 2 \times 600 \mathrm{~s}$ & 1.5 \\
& & $I$ & $1 \times 60 \mathrm{~s}, 2 \times 300 \mathrm{~s}$ & 1.5 \\
BH 222 & 1995 June 28 & $B$ & $1 \times 60 \mathrm{~s}, 2 \times 900 \mathrm{~s}$ & 1.7 \\
& & $V$ & $1 \times 60 \mathrm{~s}, 2 \times 600 \mathrm{~s}$ & 1.7 \\
& & $I$ & $1 \times 30 \mathrm{~s}, 2 \times 60 \mathrm{~s}$ & 1.7 \\
\hline
\end{tabular}

(Alter et al. 1970), BH 190 (van den Bergh \& Hagen 1975) or ESO 226-SC5 (Lauberts 1982), was first identified as an open cluster in Norma by Pismis (1959), while BH 222, located in Scorpius, was detected as an open cluster in a uniform survey of clusters in the southern Milky Way by van den Bergh \& Hagen (1975). According to Lyngå (1987), their angular diameters are about 1.0 and 2.0 arcmin, respectively. As far as we are aware, none of these two stellar groups have been previously studied.

The next section is dedicated to a brief description of the data acquisition and reduction. We confirm in Sect. 3 the existence of Pismis 23 and $\mathrm{BH} 222$ as genuine open clusters and derive their fundamental parameters. A comparison with other clusters located in similar directions is given in Sect. 4, and the final conclusions are presented in Sect. 5.

\section{Observations and reductions}

We obtained CCD BVI Johnson-Cousins images in the fields of Pismis 23 and BH 222 during two photometric nights in June 1995, with the University of Toronto Southern Observatory $0.6 \mathrm{~m}$ telescope, situated at Las Campanas Observatory (Chile). The observations were carried out using a PM $512 \times 512$ METACHROME UV coated chip, with a scale of $0.45^{\prime \prime} /$ pixel, covering a total field of about $4^{\prime} \times 4^{\prime}$. The mean seeing for the entire run was about $1.6^{\prime \prime}$ and the quality of the nights was photometric. Details of the number of observations, exposure times, and typical seeing for each object and filter, are shown in Table 1. The airmasses of the observed fields were always less than 1.35, lying just within the airmass range of the standard stars. Besides the cluster fields, we observed nightly an average of 12 standard stars from the Selected Area Standard fields (Landolt 1992). These stars were carefully selected to cover a wide range of spectral types and airmasses. Instrumental signatures were removed using bias and a combination of dome and twilight sky flats, while instrumental magnitudes were produced via point spread function (Stetson 1987). The $\mathrm{IRAF}^{1} / \mathrm{DAOPHOT}$ package was used to reduce the

\footnotetext{
${ }^{1}$ IRAF is distributed by the National Optical Astronomy Observatories, which is operated by the Association of Universities for Research in Astronomy, Inc., under contract with the National Science Foundation.
}

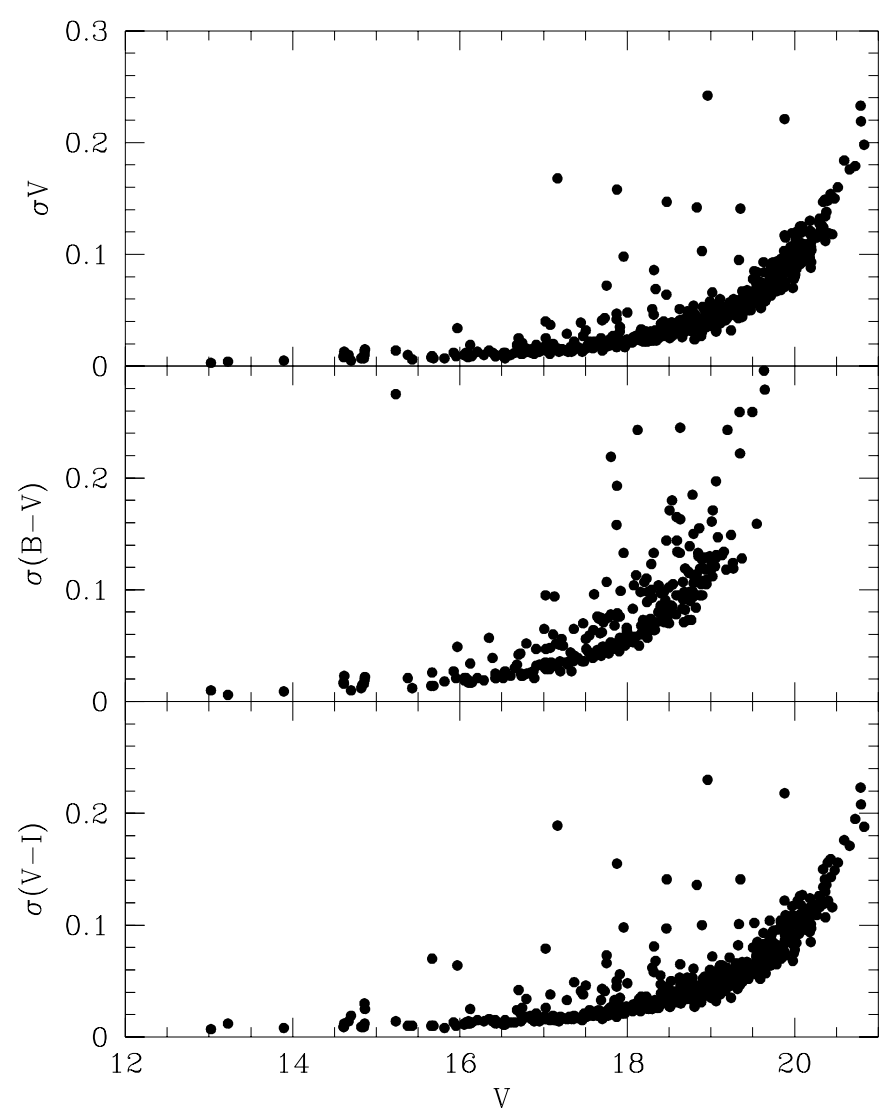

Fig. 1. Magnitude and colour photometric errors provided by DAOPHOT as a function of $V$ for the richest cluster of the sample (Pismis 23). They are typical in our sample.

observations in the standard way at the Observatorio Astronómico de la Universidad Nacional de Córdoba (Argentina). The $b, v, i$ instrumental magnitudes were obtained following the reduction procedure described in Piatti et al. (1999) and transformed into the standard system using the following relations:

$$
\begin{aligned}
& b_{j, n}=b_{1}+V+(B-V)+b_{2}(B-V)+b_{3} X_{j, n} \\
& v_{k, n}=v_{1}+V+v_{2}(B-V)+v_{3} X_{k, n} \\
& i_{l, n}=i_{1}+V-(V-I)+i_{2}(V-I)+i_{3} X_{l, n}
\end{aligned}
$$

where $V,(B-V)$ and $(V-I)$ are the standard magnitude and colours and $X$ the corresponding airmass for the $j$, $k, l$ th measured standard star. We solved Eqs. (1) to (3) for all coefficients simultaneously, for each night $n$, with the PHOTCAL package in IRAF. The mean rms errors range between $0.005 \mathrm{mag}$ and $0.020 \mathrm{mag}$ in all passbands. Figure 1 shows the trend of the photometric magnitude and colour errors with $V$ provided by DAOPHOT, for the richest cluster observed (Pismis 23). For each selected field, we generated a master table containing a running star number, the $X$ and $Y$ coordinates, the $V$ magnitudes, the $(B-V)$ and $(V-I)$ colours, the observational errors provided by the IRAF INVERTFIT task $\sigma(V)$, $\sigma(B-V)$ and $\sigma(V-I)$, and the number of observations $n$. These tables were built by combining all the independent measurements using the stand-alone DAOMATCH 
Table 2. Magnitudes and colours of stars in the field of Pismis 23.

\begin{tabular}{cccccccccc}
\hline \hline ID & $X_{\text {pixels }}$ & $Y_{\text {pixels }}$ & $V$ & $\sigma(V)$ & $B-V$ & $\sigma(B-V)$ & $V-I$ & $\sigma(V-I)$ & $n$ \\
\hline 1 & 85.486 & 1.856 & 18.793 & 0.044 & 1.025 & 0.106 & 1.578 & 0.047 & 2 \\
2 & 67.367 & 2.208 & 18.925 & 0.050 & 1.385 & 0.129 & 1.711 & 0.054 & 2 \\
3 & 391.645 & 3.210 & 17.655 & 0.019 & 1.216 & 0.042 & 1.500 & 0.020 & 2 \\
4 & 318.179 & 3.560 & 17.504 & 0.032 & 1.071 & 0.056 & 1.388 & 0.046 & 2 \\
5 & 368.840 & 4.060 & 17.550 & 0.022 & 1.144 & 0.043 & 1.401 & 0.024 & 2 \\
. &. &. &. &. &. &. &. &. &. \\
. &. &. &. &. &. &. &. &. &. \\
. &. &. &. &. &. &. &. &. &. \\
\hline
\end{tabular}

Note: Tables 2 and 3 are available in their entirety in electronic form at the CDS via anonymous ftp to cdsarc.u-strasbg.fr $(130.79 .128 .5)$ or via http://cdsweb.u-strasbg.fr/cgi-bin/qcat?J/A+A/388/179. A portion of Table 2 is shown here for guidance regarding its form and content.

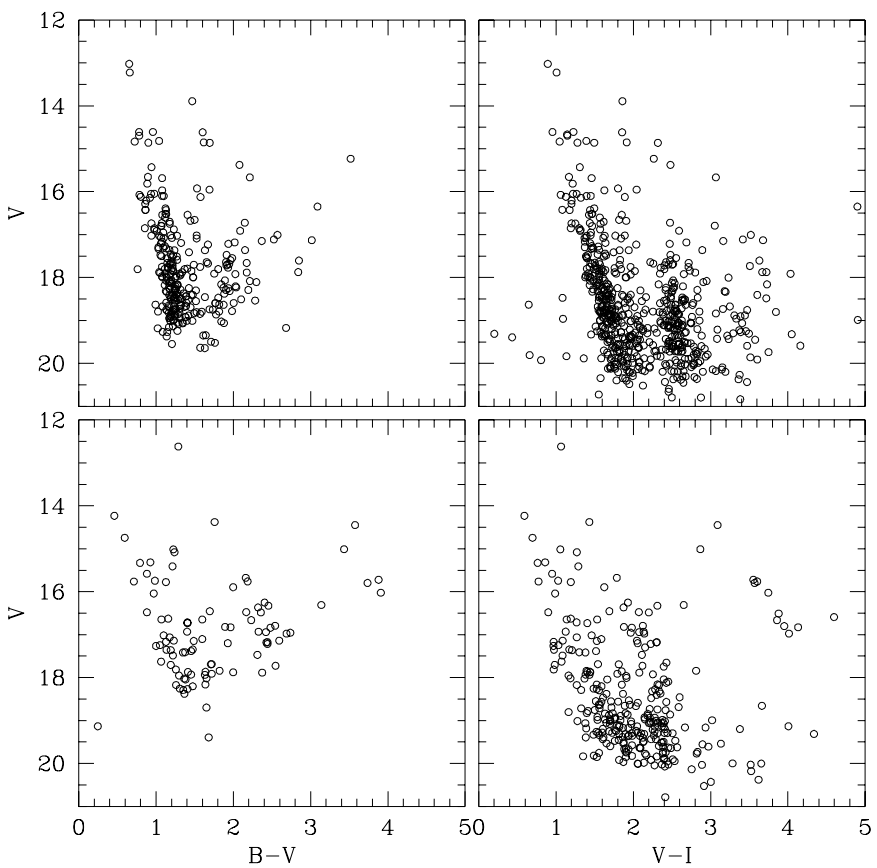

Fig. 2. $(V, B-V)$ and $(V, V-I)$ colour magnitude diagrams of stars in the field of Pismis 23 (top) and BH 222 (bottom).

and DAOMASTER programmes kindly provided by Peter Stetson. Tables 2 and 3 provide this information and are available in electronic form at the CDS. A portion of Table 2 is shown here for guidance regarding its form and content.

\section{Colour-magnitude diagram analysis}

Figure 2 shows the $(V, B-V)$ and $(V, V-I)$ CMDs containing all the measured stars for the programme fields. They reveal an important difference of $\sim 1.0-1.5$ mag in the limiting magnitudes reached between both diagrams. Since the $B$ images were typically obtained using longer exposure times, the limiting magnitude differences should be mainly caused by a strong interstellar extinction, i.e., a relatively high value of the amount of absorbed light per distance unit from the Sun in the directions towards the observed fields. While the clusters appear very bright in the $I$ images, they are scarcely visible in the $B$ ones. For BH 222 this effect turns out really dramatic, as judged from the fact that the exposure time for the deepest $I$ image was 15 times shorter than for the respective $B$ frame. Notice that, for any $V$ magnitude level, there are more brighter stars above that level in the $(V, V-I) \mathrm{CMD}$ than in the $(V, B-V)$ diagram, which constitutes an additional evidence of the high reddening that affects both fields.

The fiducial cluster sequences such as the cluster Main Sequences (MSs) and, if any, the giant and/or supergiant star sequences are not easily recognizable. The long and blue tilted star sequence corresponds in the CMDs of Pismis 23 to field MS stars belonging to the young disk (age $\sim 0.1-2.0 \mathrm{Gyr}, Z \approx 0.018-0.022$ ), to the intermediateage disk (age $\sim 4.5-7.0 \mathrm{Gyr}, Z \approx 0.008-0.015$ ), and, at its fainter portion, to the old disk populations (age $\sim 7.0$ 10.0 Gyr, $Z \approx 0.003-0.008$ ), according to the statistical results obtained by $\mathrm{Ng}$ et al. (1996) from the decomposition of CMDs of star fields observed towards Baade's Window. Following the findings of the same authors, the much less populated and nearly vertical star sequence arising slightly to the red from the bottom of the field MS $(V-I \sim 2.0)$ is composed by Horizontal Branch (HB) stars. Thus, the cluster MS should be represented by the numerous stars which define the broad and disperse sequence of redder colours, clearly seen in the $(V, V-I)$ diagram at $V-I \sim 2.4-2.8$. The MS colour dispersion is certainly caused by a high interstellar absorption. Notice that this sequence has a different lower envelope from that of the tilted blue sequence, which is an indicator that it is not composed by field MS stars (Burki \& Maeder 1973). In addition, the cluster appears to have some giant stars $(V-I \sim 3.6)$ distributed among red field stars.

The CMDs of BH 222 seem to be quite similar. The tilted blue sequence, comparable to the one in the CMDs of Pismis 23 but clearly less populous, is also composed by field MS stars. A simple comparison between the $(V$, $V-I$ ) CMDs of Pismis 23 and BH 222 could also lead 


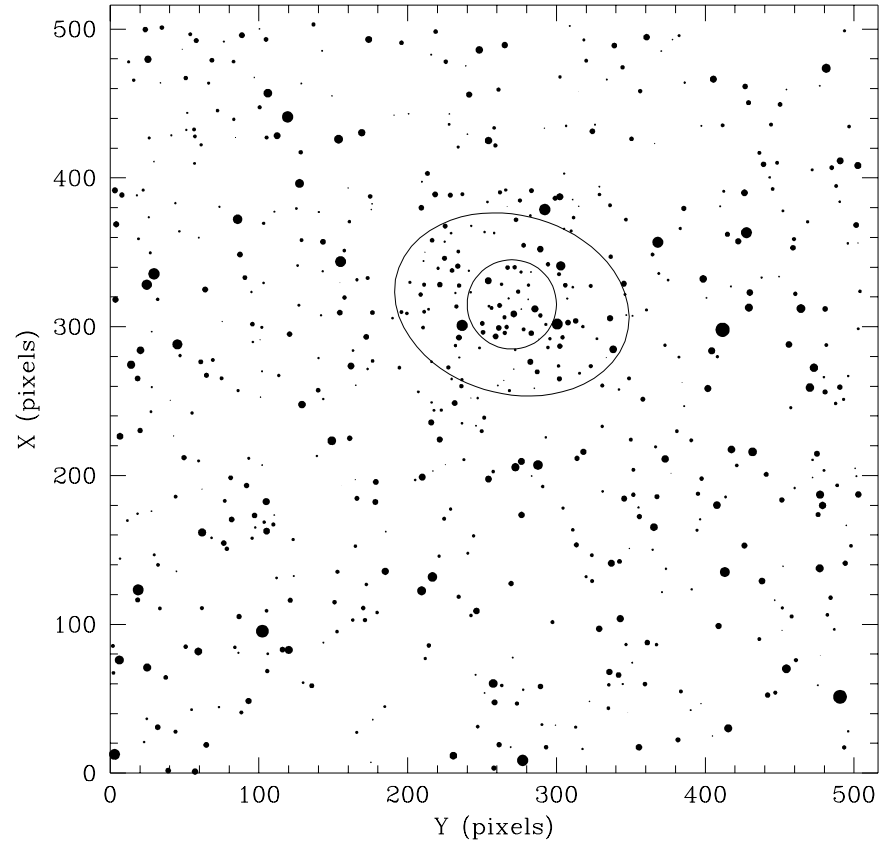

Fig. 3. Schematic finding chart for the field of Pismis 23. Two circular and elliptical extractions are also shown. North is up and East is to the left. The sizes of the plotting symbols are proportional to the $V$ magnitude of the stars.

to the conclusion that the redder sequence in the latter CMD $(V \sim 16.0-20.0, V-I \sim 2.0-2.4)$ corresponds to the cluster MS. In addition, given that the cluster was catalogued by van den Bergh \& Hagen (1975) from its appearance in their $R$ plate, we deduce that this object should also be mainly formed by the conspicuous group of bright red stars with $V \leq 17.0$ and $V-I \geq 3.4$.

\subsection{Pismis 23}

Pismis 23 was described by Ruprecht (1966) as corresponding to Trumpler (1930) class III-2m. With the aim of confirming this object as a genuine cluster, we examined the obtained images searching the region with the highest star density. The schematic finding chart built with all the measured stars in the $V$-passband (Fig. 3) reveals an excess of stars around $\left(X_{\mathrm{c}}, Y_{\mathrm{c}}\right)=(315,270)$, which we adopted as the position of the cluster centre, the sizes of the points in the figure being proportional to the visual brightness of the stars. Subsequently, we performed a circular extraction around this position and built the corresponding CMDs. In the case of an open cluster, the stars distributed within this region should define the fiducial cluster sequences, i.e., the loci in the CMDs corresponding to the mean apparent magnitudes and colours of the cluster population. We not only traced a circle of 30 (13.5 arcsec) pixels to encompass the innermost cluster region, but also drew an ellipse with minor $(b)$ and major (a) semi-axes of $60(27$ arcsec) and 80 (36 arcsec) pixels, respectively, the direction of the latter being defined by a position angle of 75 degrees. With regard to the ellipse, it was traced in order to maximize the object area

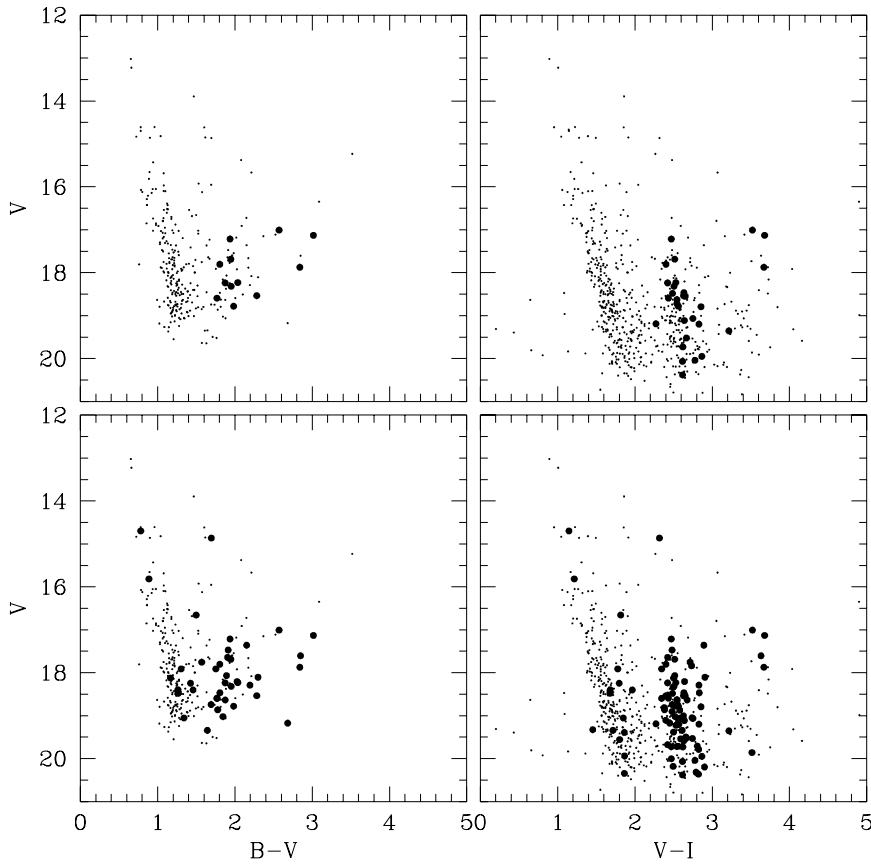

Fig. 4. $(V, B-V)$ and $(V, V-I)$ colour magnitude diagrams of stars in the field of Pismis 23: all measured stars (dots), circular extraction (filled circles, top) and elliptical extraction (filled circles, bottom).

and minimize the unavoidable field contamination. As for the position angle, it was measured anticlockwise from the $X$ axis to the $Y$ axis.

The resulting CMDs shown in Fig. 4 unambiguously demonstrate that most of the stars distributed inside the extracted areas fall along the red sequence. Stars distributed within the circular and elliptical areas are represented by filled circles in the upper and lower panels of Fig. 4, respectively, while the remaining measured stars are plotted with dots. It is important to observe that an excess of stars confined to a small region in the sky - the diameter of the extracted circle being smaller than $1 / 2$ arcmin - can develop a sequence with a different lower envelope from that of a field star sequence only if they have a common origin. This fact strongly confirms that Pismis 23 is a real cluster and that the clearly visible broad red sequence in the $(V, V-I)$ CMD corresponds to the cluster MS. Incidentally, a word of caution would be appropriate at this point: the criterion for corroborating the existence of a cluster, based on the process of looking at its extracted CMDs, can lead to uncertain results depending on several factors. Among others, we can point out the size of the extracted region, the ratio between the number of stars in the cluster as well as in the field, and the field contamination along the line of sight of the objects. The high reddening which affects the cluster - centered at $V-I \sim 2.6$ - causes the detected upper MS to appear extremely blurred in the $(V, B-V)$ CMD. Furthermore, the cluster also appears to have some red giant candidates $(V \sim 17.0-17.5, V-I \sim 3.6)$. 
Occasionally, an apparent concentration of a handful of bright stars can create the impression of an unreal cluster (see, e.g., Carraro \& Patat 1995; Piatti \& Clariá 2001a, b). Given that Pismis 23 is not clearly visible towards blue wavelenghts and shows up very bright in the near infrared, we carried out star counts in the observed field for an additional inspection of the status of the object as an open cluster. To that end, we used a red Digital Sky Survey (DSS) image of 15 arcmin on a side and centered on the object to have a wider view of the observed field. The red image was preferred over the blue one, since star counts on the latter image would have resulted in a biased indicator of the star density due to the high interstellar absorption. We identified as many stars as possible in the DSS image, using the FIND task within the stand-alone version of the DAOPHOT II package (Stetson 1994). In this respect, we were particularly cautious in the assessment of the appropriate threshold to include in the coordinate list only those objects having stellar profiles. In addition, we checked the identified stars in the DSS image, prior to counting the number of stars in the field within circles of radius $\theta_{\mathrm{obj}}$, where $\theta_{\mathrm{obj}}$ represents the semi-major axis of the cluster ellipse in Fig. 3. The choice of the circle size allowed us to compensate for the uncertainties in the defined object boundaries. We counted field stars within 100 circles selected randomly and spread throughout the DSS image, discarding those circular regions superimposed on a circle of the same dimension centered on the object. The averaged star density in the field resulted in $24 \pm 4$ stars $/ \operatorname{arcmin}^{2}$, while we counted 53 stars/arcmin ${ }^{2}$ in the cluster circle having the same area as each field. This value doubles the mean field star density and is in full agreement with the cluster reality of Pismis 23 .

Finally, we estimated the fundamental cluster parameters by fitting theoretical isochrones to the observed $(V$, $V-I$ ) CMD, making use of two different sets of isochrones, computed respectively by the Geneva (Lejeune \& Schaerer 2001, hereafter LS01) and Padova (Girardi et al. 2000, hereafter GBBC00) groups. We adopted a solar metal content $(Z=0.02)$ for the cluster, although we would have obtained similar cluster parameters if we had used adjacent metallicity values from the model grids $(Z=0.008$ or 0.04). Firstly, we adjusted the cluster MS using the position of the cluster red giants as reference. If we had neglected these giant stars, isochrones covering a wide age range could have fitted the upper MS satisfactorily, on account of its broadness and relative verticallity. In contrast, the number of isochrones that would simultaneously match both the cluster MS and the position of the red giant clump is more limited. The isochrone which best fitted the cluster sequences turned out to be that for $\log t=8.5$ of GBBC00, as is shown in Fig. 5. For the sake of comparison, we also included the isochrones for $\log t=8.3$ and 8.6 in the figure. For the fit, we assigned a higher weight to the stars distributed within the cluster ellipse (open circles) than to the remaining measured stars (dots). Figure 6 shows that the isochrone for $\log t=8.5$ calculated from the stellar evolutionary models of LS01 compares very

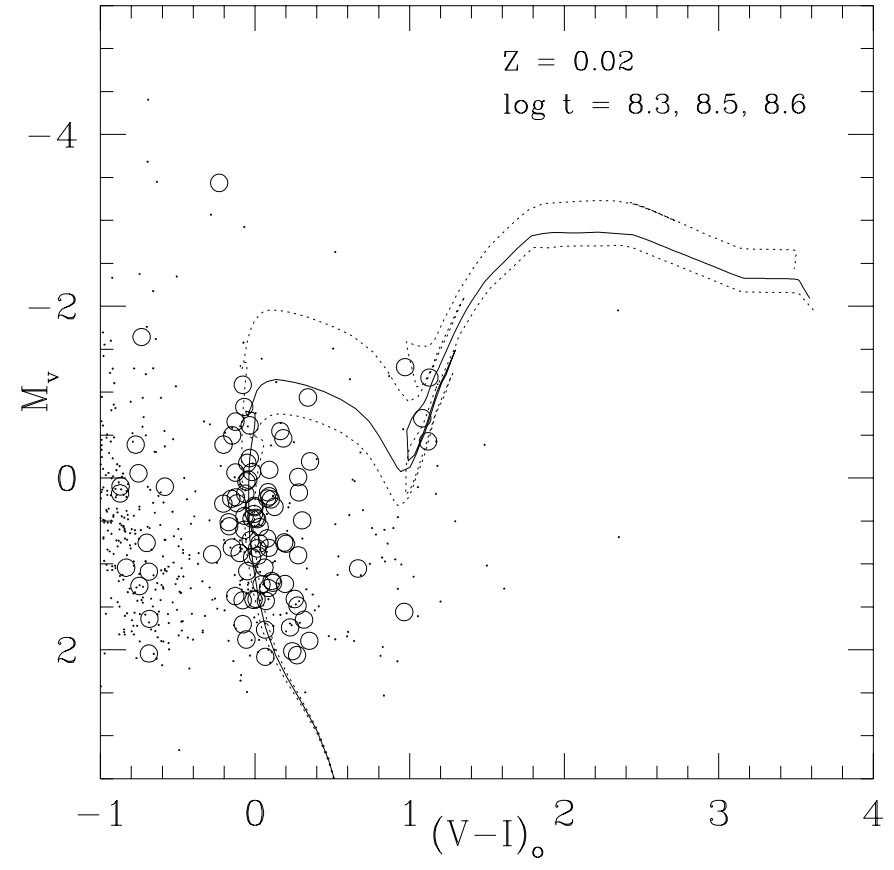

Fig. 5. $\left(M_{V},(V-I)_{\mathrm{o}}\right)$ colour magnitude diagram for Pismis 23 with the theoretical isochrones for $\log t=8.3,8.5$ and 8.6 of Girardi et al. (2000) superimposed. Stars distributed within the elliptical extraction are represented by open circles.

well with that of GBBC00. Nonetheless, since the loop at the He burning stage in the GBBC00's isochrone results slightly steeper, a more suitable fit is obtained for the giant stars from this isochrone. It should be underlined that the position in the $\left[M_{V},(V-I)_{\mathrm{o}}\right]$ plane of these giant stars is in very good agreement with the empirical locus of the giant clump region defined by Piatti et al. (1998b) for an open cluster with some hundred million years.

To estimate the $E(B-V)$ colour excess for the cluster, we used the resulting apparent distance modulus $\left(V-M_{V}=18.30 \pm 0.25\right)$ and the Zero Age Main Sequence (ZAMS) of Schmidt-Kaler (1982). Due to the fact that the cluster MS is not well defined in the $(V, B-V) \mathrm{CMD}$, the derived reddening estimate should be considered with caution. Using this value and the $E(V-I)$ colour excess obtained from the isochrone fit (the theoretical ZAMSs very tightly reproduce the empirical ZAMS of Piatti et al. 1998b), we derived a colour-to-colour $E(V-I) / E(B-V)$ ratio of $1.3 \pm 0.5$, the relatively large error being a direct consequence of the high reddening that affects the cluster. The derived ratio indicates that the interstellar absorption in the direction towards the cluster follows approximately the normal extinction law. As a result, we adopted the most frequently used values of 1.33 and 3.2 for the $E(V-I) / E(B-V)$ and total-to-selective extinction $R_{V}=A_{V} / E(B-V)$ ratios (Cousins 1978) to estimate the distance of Pismis 23. The final cluster parameters are listed in Table 4 . The uncertainty associated to the colour excesses reflects the minimum colour difference in the fitting of the ZAMS rather than the intrinsic dispersion of the cluster MS. Indeed, the differential reddening across 
Table 4. Adopted cluster fundamental parameters.

\begin{tabular}{lccccc}
\hline \hline Cluster & $E(B-V)$ & $E(V-I)$ & $V_{\mathrm{o}}-M_{V}$ & $\begin{array}{c}d \\
(\mathrm{kpc})\end{array}$ & $\begin{array}{c}\text { Age } \\
(\mathrm{Myr})\end{array}$ \\
\hline Pismis 23 & $2.0 \pm 0.1:$ & $2.6 \pm 0.1$ & $12.0 \pm 0.5$ & $2.6 \pm 0.6$ & $300 \pm 100$ \\
BH 222 & - & $2.4 \pm 0.2$ & $13.9 \pm 0.5$ & $6.0 \pm 2.7$ & $60 \pm 30$ \\
\hline
\end{tabular}

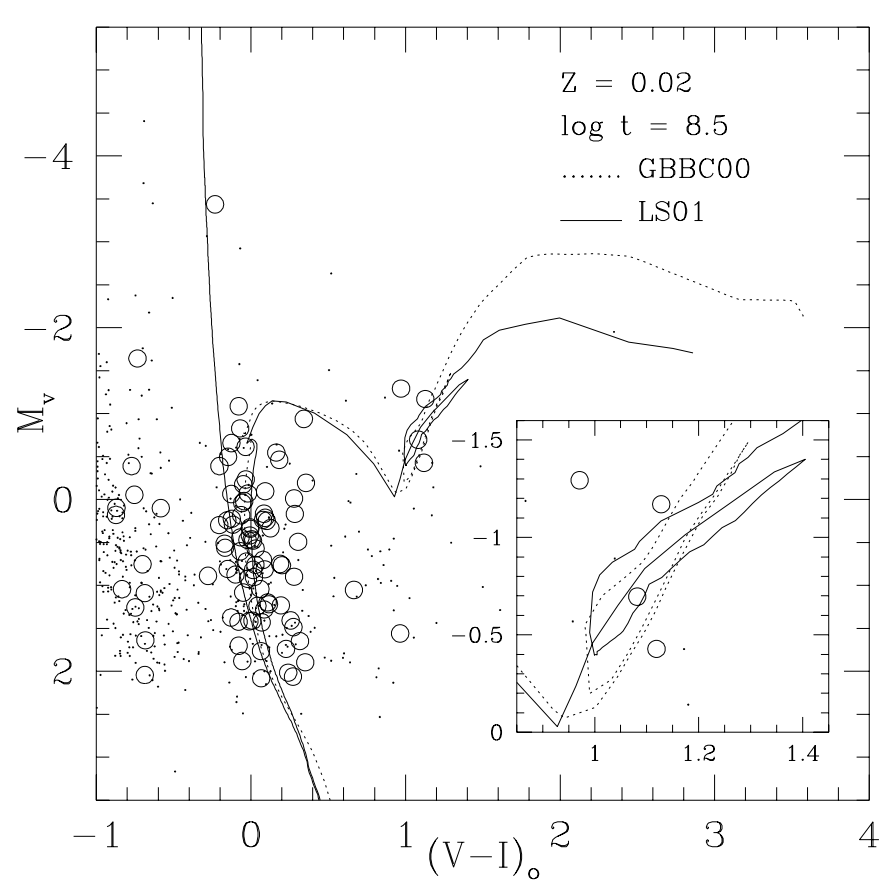

Fig. 6. $\left(M_{V},(V-I)_{\mathrm{o}}\right)$ colour magnitude diagram for Pismis 23 with the Zero Age Main Sequence and the theoretical isochrones for $\log t=8.5$ computed by Girardi et al. (2000) (GBBC00, dotted line) and Lejeune \& Schaerer (2001) (LS01, solid line) superimposed. An enlargement of the giant clump region is shown at the lower-right corner. Symbols are the same as in Fig. 5.
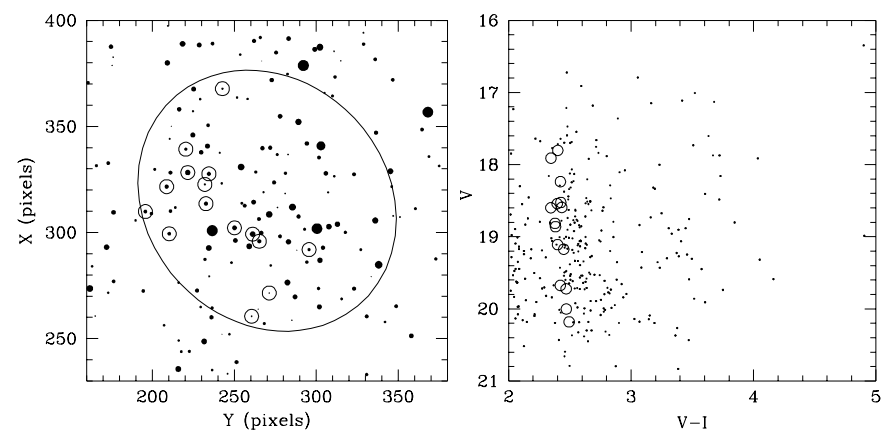

Fig. 7. Schematic finding chart (left panel) and $(V, V-I)$ CMD (right panel) for stars measured in the field of Pismis 23. North is up and East is to the left in the finding chart. Open circles represent stars located within the cluster ellipse and distributed along the lower envelope of the extracted MS.

the cluster could be at least of $\Delta E(B-V) \sim 0.2$ mag. As Fig. 7 shows, the stars distributed within the cluster ellipse and along the lower envelope of the extracted MS (open circles) appear preferably located towards the

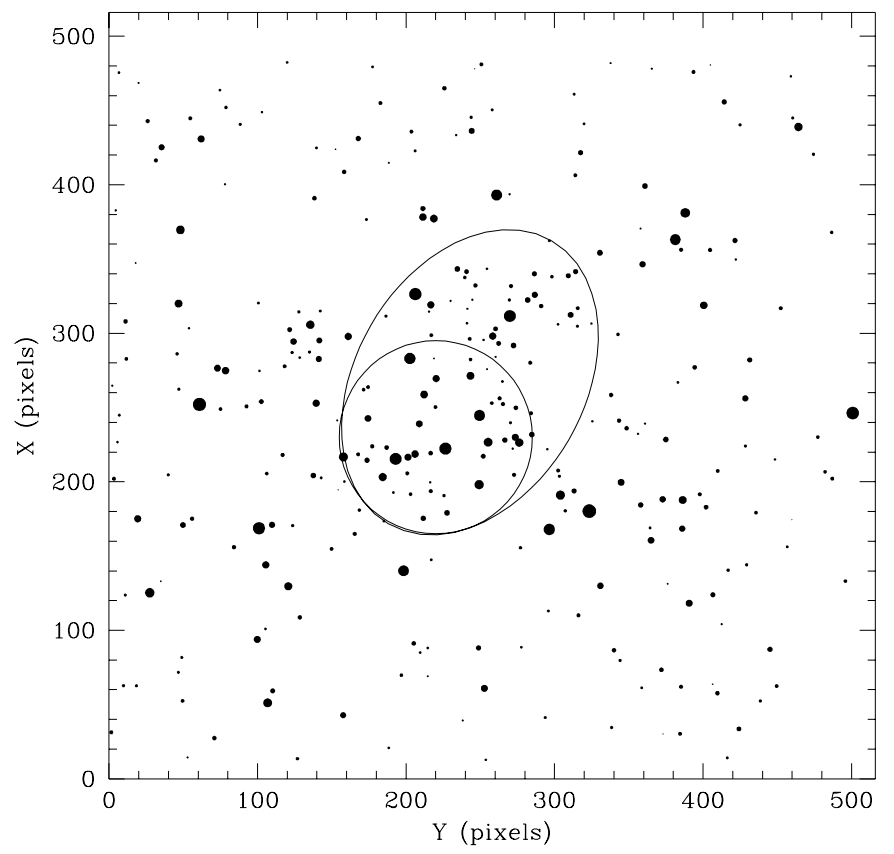

Fig. 8. Schematic finding chart for the field of BH 222. Two circular and elliptical extractions are also shown. North is up and East is to the left. The sizes of the plotting symbols are proportional to the $I$ magnitude of the stars.

eastern - southeastern half of the ellipse. The distance error was computed with the expression: $\sigma(d)=0.46 \times[\sigma$ $\left.\left(V-M_{V}\right)+3.2 \times \sigma(E(B-V))\right] \times d$, where $\sigma\left(V-M_{V}\right)$ and $\sigma(E(B-V))$ represent the estimated errors in $V-M_{V}$ and $E(B-V)$, respectively.

\section{2. $\mathrm{BH} 222$}

We employed a similar strategy to analyze the CMDs of BH 222, our goals being the confirmation that the object consists of a physical system and the estimation of its age, reddening and distance from the Sun. Taking into account the noticeable contrast in the number and brightness of the detected stars between the blue and near-infrared images, we first carried out circular and elliptical extractions of the most populated regions on the deepest $I$ frame and built the corresponding CMDs. Figure 8 shows the obtained schematic finding chart for all the stars with at least one measurement in the $I$-passband. The sizes of the filled circles are proportional to the $I$ magnitudes of the stars. Accordingly, this chart roughly reproduces the observed field as it is seen in the direct CCD $I$ image. The positions and dimensions of the circle and the ellipse 


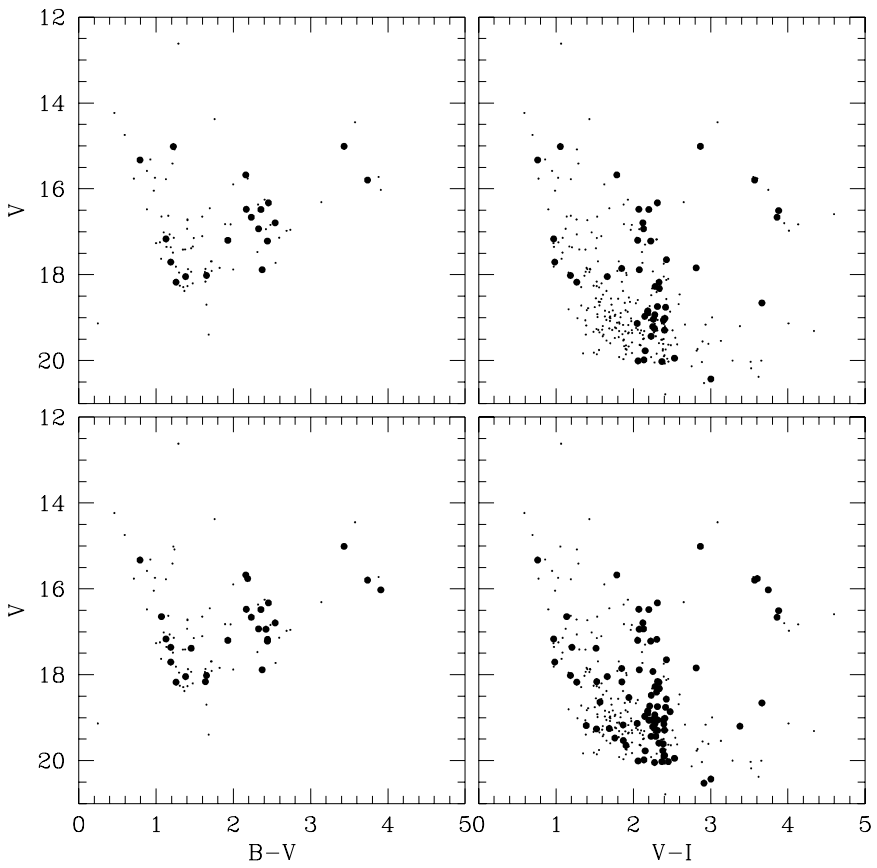

Fig. 9. $(V, B-V)$ and $(V, V-I)$ colour magnitude diagrams of stars in the field of BH 222: all measured stars (dots), circular extraction (filled circles, top) and elliptical extraction (filled circles, bottom).

drawn in Fig. 8 were chosen as an attempt to delineate the nuclear parts of the cluster and its boundary. Figure 9 reproduces the $(V, B-V)$ and $(V, V-I)$ CMDs of the stars observed in the field of $\mathrm{BH} 222$ and shows the loci of the stars (filled circles) distributed within the circular (top panels) and elliptical (bottom panels) extractions, respectively. Even though the $(V, B-V)$ CMD proves to be barely useful to delineate the morphology of some sequences of stars, the $(V, V-I)$ CMD undoubtedly reveals the main cluster features. The distribution of the extracted stars in the $(V, V-I)$ CMD closely resembles that of a vertical MS with very red luminous stars, typically red supergiants (RSGs), of a young cluster. Several field stars distributed along the blue star sequence also contaminate the extracted CMDs.

Aside from a clear field star contamination, the cluster MS also appears to suffer from a noticeable differential extinction. As Fig. 9 shows, the cluster MS presents an small colour offset at $V \sim 18 \mathrm{mag}$, which causes the brighter stars to appear slightly bluer. Stars belonging to both parts of this "cracked" MS are mostly supposed to be cluster stars, a fact from which we believe that this apparent break is caused by a non-uniform interstellar absorption across the cluster field. This conclusion is illustrated in Fig. 10, wherein we represent with open circles stars located in the upper extracted MS. Notice that these stars are predominantly placed towards the eastern side of the cluster. The mean colour difference between both MS segments resulted in $\Delta(V-I) \sim 0.2$ mag. Therefore, to determine the fundamental cluster parameters, we adopted an intermediate position between them when fitting the
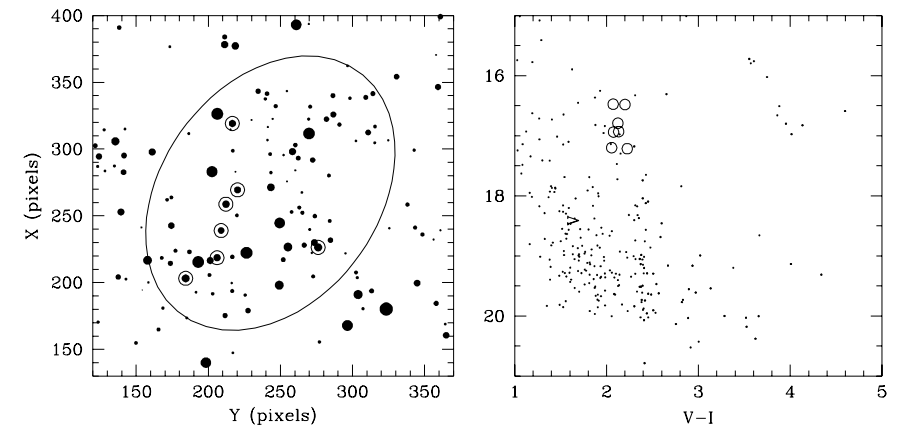

Fig. 10. Schematic finding chart (left panel) and $(V, V-I)$ CMD (right panel) for stars measured in the field of BH 222. North is up and East is to the left in the finding chart. Open circles represent stars located within the cluster ellipse and distributed along the upper extracted cluster MS.

ZAMS to the extracted CMD, and assumed that a similar difference in the $V-I$ colour could also affect the positions of the RSGs. Recent studies on highly reddened young open clusters have also shown that cluster MSs, although vertical and well-defined, distinguish themselves from their broadness and dispersion. This is the case of Weslertund 1 (Piatti et al. 1998a) and of a recently discovered star cluster embedded in the Galactic plane (Nakaya et al. 2001), which are affected by visual absorptions of $A_{V} \sim 13.0$ and 11.2, respectively. Finally, we performed a star count in the field of $\mathrm{BH} 222$ following the same precepts as for Pismis 23 and found a mean density of $17 \pm 3$ stars $/ \operatorname{arcmin}^{2}$, while we counted $40 \mathrm{stars} / \operatorname{arcmin}^{2}$ in the surrounding field. These values, read with the proper caution since they were computed from a nonuniform reddened field, corroborate the physical existence of BH 222.

To estimate the fundamental cluster parameters, we fitted theoretical isochrones following the procedure applied for Pismis 23. As for the Padova group, we used the isochrones computed by Salasnich et al. (2000, hereafter SGWC00), since they extended those of GBBC00 towards younger ages. A solar metal content was assumed for BH 222 and special attention was devoted to the morphology of the RSG evolutionary phase. The result of the fit is shown in Fig. 11, where three isochrones for $\log t=7.5$, 7.8 and 8.0 calculated by SGWC00 are superimposed for comparison purposes. Regardless of the effect of differential reddening across the cluster field, the isochrone for log $t=7.8$ satisfactorily fits the MS cluster stars. However, although there also exists a tight agreement between the positions of the RSGs and the theoretical red giant branch (see Fig. 12), the latter results $\sim 0.6 \mathrm{mag}$ bluer than the RSGs. For this reason, we considered the cluster MS and the bottom of the He burning phase to estimate the mean cluster reddening and apparent distance modulus, respectively. The $V-I$ colour difference between RSGs and the isochrones probably arises not only from the effect of differential reddening, but also from the input physics and evolutionary codes used, since it is nearly 3 times larger than the colour difference $(\Delta(V-I) \sim 0.2)$ obtained from 


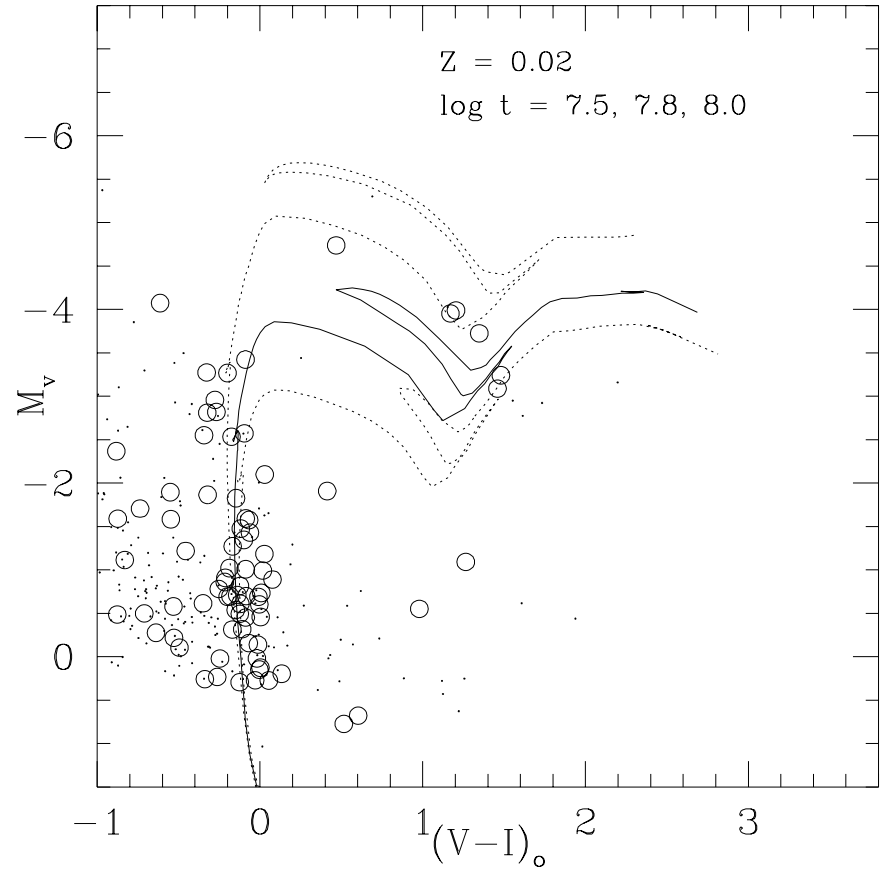

Fig. 11. $\left(M_{V},(V-I)_{\mathrm{o}}\right)$ colour-magnitude diagram for BH 222 with the theoretical isochrones for $\log t=7.5,7.8$ and 8.0 of Salasnich et al. (2000) superimposed. Stars distributed within the elliptical extraction are represented by open circles (see text for details).

the upper and lower cluster MS. In Fig. 12 the isochrones have been shifted to obtain the best fit with the observed points. As with Pismis 23, we achieved a slightly better fit using the isochrone for $\log t=7.8$ computed by SGWC00, instead of that of LS01. Figure 12 shows how the RSG phase in the isochrone of LS01 turns out to be redder than the corresponding loop in the SGWC00's isochrone. Likewise, we did not find a noticeable difference in the fit if isochrones with high mass loss for massive stars were used. The final cluster parameters are listed in Table 4 together with their errors, which were estimated as for Pismis 23.

\section{Discussion}

The quantities listed in Table 4 are the first estimates, as far as we are aware, of the fundamental properties for Pismis 23 and BH 222. The reality of this latter object as an open cluster was suggested by van den Bergh \& Hagen (1975), who also mentioned that the cluster could probably be embedded in a nebulosity. The resulting reddening and distance estimates place these two objects among the most reddened and distant open clusters located in the direction towards the Galactic centre, a result which is illustrated in Fig. 13. With the help of the WEBDA Open Cluster Database (see, http://obswww.unige.ch/webda/), we selected a list of clusters distributed within a solid angle centered at the midst of both clusters, defined by $332^{\circ} \leq l \leq 352^{\circ}$ and $-2.5^{\circ} \leq b \leq 2.5^{\circ}$, and with known values of their colour excesses and distances. We present in Table 5

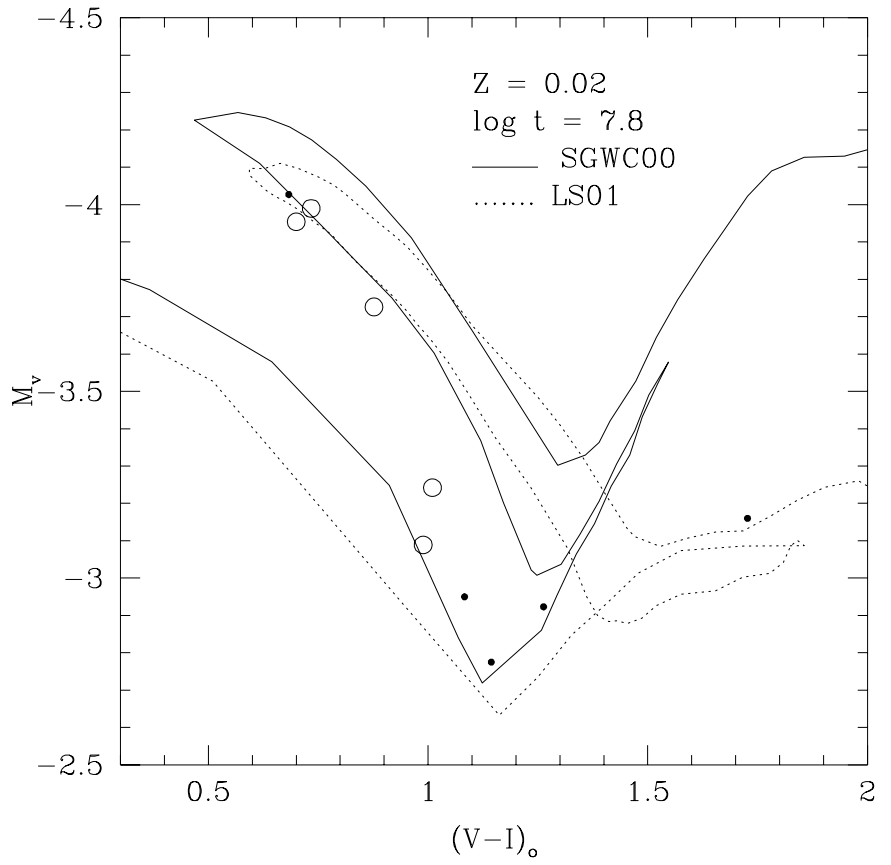

Fig. 12. Giant clump region of the $\left(M_{V},(V-I)_{\mathrm{o}}\right)$ colour magnitude diagram for $\mathrm{BH} 222$ with the theoretical isochrones for $\log t=7.8$ computed by Salasnich et al. (2000) (SGWC00, solid line) and Lejeune \& Schaerer (2001) (LS01, dotted line) superimposed. The isochrones were shifted to obtain the best match with giant clump stars. Symbols are the same as in Fig. 11.

the final cluster list with the values of the adopted parameters and corresponding references, having averaged the involved quantities when two or more references were used. We show in the upper-right panel of Fig. 13 that $\mathrm{BH} 222$ is the most distant cluster and that only three clusters (Ruprecht 119, NGC 6216 and Havlen-Moffat 1) are more distant than Pismis 23. In the figure we represented the selected clusters with open circles and the two studied clusters with stars. The distances estimated for Pismis 23 and BH 222 placed them beyond the Sagittarius arm, close to the direction where this arm probably bifurcates into two arms (Vogt \& Moffat 1975; Georgelin \& Georgelin 1976). In particular, BH 222 is projected against the HII region RCW 123 (Rodgers et al. 1960; Georgelin \& Georgelin 1970). It must also be underscored that, with the sole exception of Westerlund $1(E(B-V)=4.3$, Piatti et al. 1998a), Pismis 23 and BH 222 are among the clusters affected by the highest $E(B-V)$ colour excesses (lower panels). We used $E(B-V)=1.8$ for $\mathrm{BH} 222$, as derived from the $E(V-I)$ value. Finally, the lower-right panel of Fig. 13 suggests that, the farther the clusters from the Sun, the larger the range of the $E(B-V)$ colour excesses, although at larger distances higher reddening values prevail. In this context, it would be worthwhile to know the metallicities of these clusters, which cover a wide baseline in Galactocentric distances. Such knowledge could be used to determine the chemical abundance gradient along their line of sight and compare it with those obtained for other directions, with the aim of investigating whether the 


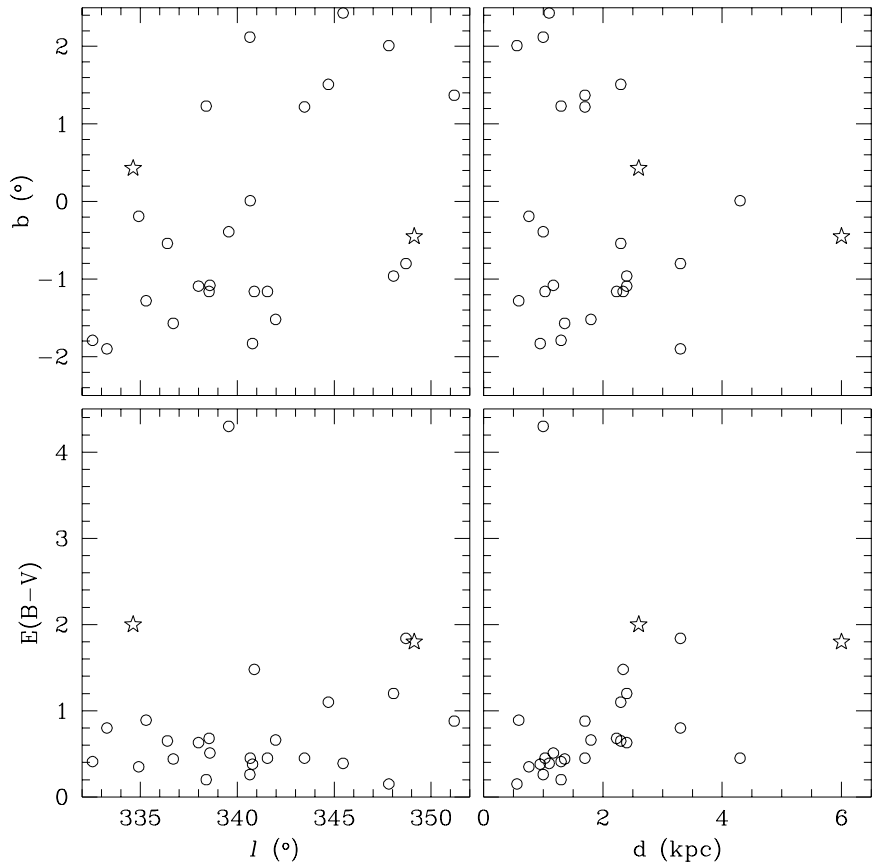

Fig. 13. Relationship between the Galactic longitude and latitude, the distance from the Sun and the reddening of open clusters with known fundamental parameters (open circles) located in the direction towards Pismis 23 and BH 222 (open stars) (see Sect. 4 for details).

radial abundance gradient shows some dependence on the Galactic longitude across the Galactic disk.

\section{Conclusions}

We provide CCD BVI Johnson-Cousins photometry for the southern stellar groups Pismis 23 and $\mathrm{BH} 222$, which are described as open clusters in several catalogues (Alter et al. 1970; Lyngå 1987; Lauberts 1982). As far as we are aware, these two stellar groups, located in Norma and Scorpius, respectively, close to the direction towards the Galactic centre, have not been previously studied. The observed $(V, B-V)$ and $(V, V-I)$ CMDs in the field of Pismis 23 reveal the presence of an open cluster MS, as well as a few red giant candidates. We also found that the star density in the cluster field doubles the mean field star density, in full agreement with the cluster reality of Pismis 23. The average $E(B-V) / E(V-I)$ ratio derived for this cluster was found to be $1.3 \pm 0.5$, in very good agreement with the value generally adopted for the normal extinction law. The present photometric data indicate that Pismis 23, located at a distance of $2.6 \pm 0.6 \mathrm{kpc}$ from the Sun, is a moderately young open cluster with an age of $300 \pm 100$ Myr.

The distribution of comparatively bright red stars in the $(V, V-I)$ CMD of $\mathrm{BH} 222$, aside from a vertical MS, closely resembles that of very luminous objects, typically red supergiant stars, of a young cluster. BH 222, projected against the HII region RCW 123, is affected by a high interstellar absorption, the colour excess $E(V-I)$
Table 5. Fundamental parameters for clusters located in the direction towards Pismis 23 and $\mathrm{BH} 222$.

\begin{tabular}{lccccc}
\hline \hline Cluster & $\begin{array}{c}l \\
\left(^{\circ}\right)\end{array}$ & $\begin{array}{c}b \\
\left.{ }^{\circ}\right)\end{array}$ & $E(B-V)$ & $\begin{array}{c}d \\
(\mathrm{kpc})\end{array}$ & Ref. \\
\hline Ruprecht 118 & 332.54 & -1.79 & 0.41 & 1.30 & 1 \\
Ruprecht 119 & 333.28 & -1.90 & 0.80 & 3.30 & 2 \\
NGC 6134 & 334.92 & -0.19 & 0.35 & 0.76 & 3 \\
NGC 6167 & 335.30 & -1.28 & 0.89 & 0.59 & 4 \\
Ruprecht 120 & 336.40 & -0.54 & 0.65 & 2.30 & 5 \\
NGC 6193 & 336.70 & -1.57 & 0.44 & 1.36 & 1 \\
NGC 6200 & 338.00 & -1.09 & 0.63 & 2.40 & 6 \\
NGC 6178 & 338.40 & 1.23 & 0.20 & 1.30 & 7 \\
Hogg 22 & 338.55 & -1.16 & 0.68 & 2.23 & 8 \\
NGC 6204 & 338.59 & -1.08 & 0.51 & 1.17 & 8 \\
Westerlund 1 & 339.56 & -0.39 & 4.30 & 1.00 & 9 \\
NGC 6192 & 340.65 & 2.12 & 0.26 & 1.00 & 10 \\
NGC 6216 & 340.67 & 0.01 & 0.45 & 4.30 & 7 \\
NGC 6250 & 340.79 & -1.83 & 0.38 & 0.95 & 4 \\
Lyngå 14 & 340.88 & -1.16 & 1.48 & 2.34 & 4 \\
NGC 6249 & 341.56 & -1.16 & 0.45 & 1.03 & 1 \\
NGC 6259 & 341.98 & -1.52 & 0.66 & 1.80 & 11,12 \\
NGC 6231 & 343.47 & 1.22 & 0.45 & 1.70 & $13,14,15$ \\
Trumpler 24 & 344.70 & 1.51 & 1.10 & 2.30 & 16 \\
NGC 6242 & 345.46 & 2.43 & 0.39 & 1.10 & $1,13,17$ \\
NGC 6281 & 347.82 & 2.01 & 0.15 & 0.56 & 18 \\
NGC 6318 & 348.06 & -0.96 & 1.20 & 2.40 & 2 \\
Havlen-Moffat 1 & 348.70 & -0.8 & 1.84 & 3.30 & 19 \\
Bochum 13 & 351.19 & 1.37 & 0.88 & 1.70 & 4 \\
\hline & & & & & \\
\hline
\end{tabular}

References: 1) Moffat \& Vogt (1973); 2) Piatti et al. (2000a); 3) Clariá \& Mermilliod (1992); 4) Moffat \& Vogt (1975); 5) Piatti et al. (1999); 6) Fitzgerald et al. (1977); 7) Piatti et al. (2000b); 8) Forbes \& Short (1996); 9) Piatti et al. (1998a); 10) King (1987); 11) Hawarden (1974); 12) Anthony-Twarog \& Twarog (1987); 13) Meynet et al. (1993); 14) Balona \& Laney (1995); 15) Perry et al. (1991); 16) Heske \& Wendker (1984); 17) Battinelli \& Capuzzo-Dolcetta (1991); 18) Feinstein \& Forte (1974); 19) Vázquez \& Baume (2001).

being $2.4 \pm 0.2$. We also found evidence that the interstellar material in front of the cluster is non uniformlydistributed. Based on the theoretical isochrones that are in better agreement with our data, we estimate a distance from the Sun of $6.0 \pm 2.7 \mathrm{kpc}$ and an age of $60 \pm 30 \mathrm{Myr}$, assuming solar metallicity for BH 222 .

The resulting parameters for Pismis 23 and $\mathrm{BH} 222$ place these two objects among the most reddened and distant open clusters known in the direction towards the Galactic centre. In particular, the distances derived place these two clusters beyond the Sagittarius arm. It would be important to derive the cluster metallicities in order to improve our knowledge of the radial metal abundance gradient and the age-metallicity relationship for the disk of our Galaxy, using a longer baseline in Galactocentric distances.

Acknowledgements. We have used DSS images for the analysis. The DSS was produced at the Space Telescope Science Institute under U.S. Government grant NAG W-2166. 
The images of these surveys are based on photographic data obtained using the Oschin Schmidt Telescope on Palomar Mountain and the UK Schmidt Telescope. We wish to thank the Director, staff and technicians of Las Campanas Observatory for the allocation of observing time and for their kind assistance and hospitality during the observing runs. We also thank the anonymous referee for his valuable comments and suggestions. We gratefully acknowledge financial support from the Argentinian institutions CONICET, SECYT (Universidad Nacional de Córdoba), and AGENCIA CÓRDOBA CIENCIA.

\section{References}

Alter, G., Ruprecht, J., \& Vanisek, J. 1970, Catalogue of Star Clusters and Associations, ed. G. Alter, B. Balázs, \& J. Ruprecht (Akademiai Kiado, Budapest)

Anthony-Twarog, B. J., \& Twarog, B. A. 1987, AJ, 94, 1222

Balona, L. A., \& Laney, C. D. 1995, MNRAS, 276, 627

Battinelli, P., \& Capuzzo-Dolcetta, R. 1991, MNRAS, 249, 76

Burki, G., \& Maeder, A. 1973, A\&A, 25, 71

Carraro, G., \& Patat, F. 1995, MNRAS, 276, 563

Clariá, J. J., \& Mermilliod, J.-C. 1992, A\&AS, 95, 429

Cousins, A. W. J. 1978, Mon. Not. R. Astron. Soc. S. Afr., 37,62

Feinstein, A., \& Forte, J. C. 1974, PASP, 86, 284

Fitzgerald, P. M., Jackson, P. D., \& Moffat, A. F. J. 1977, Observatory, 97, 129

Forbes, D., \& Short, S. 1996, AJ, 111, 1609

Georgelin, Y. P., \& Georgelin, Y. M. 1970, A\&AS, 3, 1

Georgelin, Y. M., \& Georgelin, Y. P. 1976, A\&A, 49, 57

Girardi, L., Bressan, A., Bertelli, G., \& Chiosi, C. 2000, A\&AS, 141,371

Hawarden, T. G. 1974, MNRAS, 169, 539

Heske, A., \& Wendker, H. J. 1984, A\&AS, 57, 205

Janes, K. A., \& Adler, D. 1982, ApJS, 49, 425

Janes, K. A., \& Phelps, R. 1994, AJ, 108, 1773

King, D. F. 1987, Observatory, 107, 107

Landolt, A. U. 1992, AJ, 104, 340

Lauberts, A. 1982, The ESO/Uppsala Survey of the ESO(B) Atlas, European Southern Observatory, Garching

Lejeune, T., \& Schaerer, D. 2001, A\&A, 366, 538
Lyngå, G. 1965, Medd. fran Lunds Observatorium, Ser. II, No. 143

Lyngå, G. 1987, Catalogue of Open Cluster Data, Strasbourg: Centre de Données Stellaires

Meynet, G., Mermilliod, J.-C., \& Maeder, A. 1993, A\&AS, 98, 477

Moffat, A. F. J., \& Vogt, N. 1973, A\&AS, 10, 135

Moffat, A. F. J., \& Vogt, N. 1975, A\&AS, 20, 155

Nayaka, H., Watanabe, M., Ando, M., et al. 2001, AJ, 122, 876

Ng, Y. K., Bertelli, G., Chiosi, C., \& Bressan, A. 1996, A\&A, 310, 771

Perry, C. L., Hill, G., \& Christoudou, D. M. 1991, A\&AS, 90, 195

Phelps, R., \& Janes, K. A. 1993, AJ, 106, 1870

Piatti, A. E., Bica, E., \& Clariá, J. J. 1998a, A\&AS, 127, 423

Piatti, A. E., Bica, E., \& Clariá, J. J. 2000a, A\&A, 362, 959

Piatti, A. E., \& Clariá, J. J. 2001a, A\&A, 379, 453

Piatti, A. E., \& Clariá, J. J. 2001b, A\&A, 370, 931

Piatti, A. E., Clariá, J. J., \& Abadi, M. G. 1995, AJ, 110, 2813

Piatti, A. E., Clariá, J. J., \& Bica, E. 1998b, ApJS, 116, 263

Piatti, A. E., Clariá, J. J., \& Bica, E. 1999, MNRAS, 303, 65

Piatti, A. E., Clariá, J. J., \& Bica, E. 2000b, A\&A, 360, 529

Pismis, P. 1959, Bol. Tonantzintla y Tacubaya, 18, 37

Rodgers, A. W., Campbell, C. T., Whiteoak, J. B., et al. 1960, An Atlas of H-alpha emission in the Southern Milky Way (Mt. Stromlo Obs., Canberra)

Ruprecht, J. 1966, Bull. Astron. Inst. Czech., 17, 33

Salasnich, B., Girardi, L., Weiss, A., \& Chiosi, C. 2000, A\&A, 361,1023

Sandage, A. R. 1988, in Calibration of Stellar Ages, Schenectady USA, ed. A. G. D. Phillip, \& L. Davis, 43

Schmidt-Kaler, Th. 1982, in Landolt-Börnstein. Numerical Data and Functional Relationships in Science and Technology, New Ser., group VI, vol. 2b, ed. K. Schaifers, \& H. H. Voigt (Berlin, Springer Verlag)

Stetson, P. B. 1987, PASP, 99, 191

Stetson, P. B. 1994, PASP, 106, 250

Trumpler, R. J. 1930, Lick Obs. Bull., 14, 154

Twarog, B. A., Ashman, K. M., \& Anthony-Twarog, B. J. 1997, AJ, 114, 2556

Vázquez, R. A., \& Baume, G. 2001, A\&A, 371, 908

van den Berg, S., \& Hagen, G. L. 1975, AJ, 80, 11

Vogt, N., \& Moffat, A. F. J. 1975, A\&A, 39, 477 